\title{
Investigar el cuidado comunitario de la vida a través de las artes plásticas. Una experiencia en contexto rural
}

\author{
Investigate the community care for life through the plastic arts. An experience in rural context
}

Beatriz Elena Arias-López ${ }^{*}$ orcid.org/0000-0002-3326-0402

Laura Antonia Coral-Velásquez² orcid.org/0000-0002-6771-6404

1 Facultad de Enfermería, Universidad de Antioquia. Medellín, Colombia

2 Maestra en Artes Plásticas y Visuales. Cundinamarca, Colombia contexto rural. Univ. Salud. 2017;19(1):75-84. DoI: http://dx.doi.org/10.22267/rus.171901.71

\section{Resumen}

Objetivo: Presentar los principales aprendizajes de la incorporación del arte como mediador, en una investigación sobre memorias de cuidado campesino alrededor del alimento y la producción agrícola. Materiales y métodos: Investigación cualitativa, orientada por la Investigación - Acción - Participativa (IAP), que desarrolló entrevistas, observación participante y cartografía social, en diálogo con técnicas artísticas para la producción de datos y para la difusión de hallazgos. Resultados: Se describen los aportes del arte a la construcción de las memorias mediante la incorporación de un objeto con fuerte carga simbólica, para materializarlas y favorecer su difusión concretada en la obra plástica "Memorias sobre machetes". Discusión. Aunque no fue su intención, la experiencia se acerca con el movimiento emergente de Investigación basada en el arte, lo que se expresó en rupturas y articulaciones desde el punto de vista de la técnica artística, del proceso investigativo y el diálogo transdisciplinar arte-enfermería. Conclusiones. 1) Fortalecimiento del patrón de conocimiento estético en enfermería, 2) Fortalecimiento de la investigación cualitativa desde los aportes del arte, 3) Reconocimiento de las voces que se encuentran en las márgenes del sistema social y 4) Aportes estratégicos del arte para la construcción de memorias.

Palabras clave: Arte; Investigación en Enfermería; Investigación Cualitativa; población rural; Mujeres. (Fuente: DeCS, Bireme).

\begin{abstract}
Objective: To present the main learnings of the incorporation of art as a mediator in a research about reports of farmer care around food and agricultural production. Materials and methods: A qualitative research oriented by Participatory Action Research (PAR) was conducted. Interviews, participant observation and social mapping, in dialogue with artistic techniques for the production of data and the dissemination of findings, were developed. Results: The contributions of art to the construction of the reports are described through the incorporation of an object with a strong symbolic load, to be materialized and encourage their dissemination embodied in the sculpture "Memorias sobre machetes". Discussion: Although it was not its intention, the experience is close to the emerging
\end{abstract}


movement of research based on the art, which was expressed in breaks and joints from the artistic technique point of view, the investigative process and the transdisciplinary dialog between art and nursing. Conclusions: 1) Strengthening of the pattern of aesthetic knowledge in nursing, 2) Strengthening of the qualitative research from the contributions of art, 3) Recognition of the voices that are found in the margins of the social system and 4) Strategic art Inputs for the construction of reports.

Keywords: Art; Nursing Research; Qualitative Research; rural population; women. (Source: DeCS, Bireme).

\section{Introducción}

Este artículo recoge los resultados derivados de un diálogo intencionadamente transdisciplinar que confluyeron en un proyecto de investigación denominado: "Construcción de una memoria femenina, colectiva y campesina sobre el alimento: saberes y prácticas de cuidado familiar y comunitario" (Proyecto en alianza entre la Facultad de Enfermería de la Universidad de Antioquia y la Asociación Campesina de Antioquia) y un proyecto artístico para optar el título de Maestra en Artes Plásticas y visuales (Universidad Distrital de Bogotá) denominado "Memorias sobre machetes". La articulación de ambos procesos generó una serie de aprendizajes de orden teórico, metodológico y práctico en la investigación y acción en salud, involucrando el arte como mediación. La experiencia se llevó a cabo en un municipio del oriente antioqueño entre 2014 y 2015.

La articulación del arte en este proceso de investigación sobre cuidado familiar $y$ comunitario con mujeres de una zona rural, se alimenta de los debates que emergieron en la segunda mitad del siglo XX alrededor de la investigación cualitativa $\mathrm{y}$ otras formas de indagación no positivistas $^{1}$, dirigidas al reconocimiento de "verdades personales" y al acercamiento sensible a los sujetos, que los modelos objetivadores habían relegado y que se plasman en aquellos que emergieron en los años setenta en el mundo anglosajón bajo diversas denominaciones, agrupados en la llamada investigación basada en el arte (o ABR por sus siglas en inglés: Arts-Based Research) ${ }^{2,3}$. Bajo esta lente, el arte es considerado un mediador que activa, inspira, despierta y logra plasmar los resultados de la investigación de manera emocional y evocadora, que además fortalece su potencial estético y político, no sólo para quienes participan directamente en el proceso creativo investigativo, sino además para otra diversidad de audiencias, espectadores y actores sociales. La vinculación de la investigación con las artes interroga epistemológica y metodológicamente las formas convencionales de investigación, a la vez que introduce expresiones y procedimientos artísticos en su proceso ${ }^{4}$.

En el caso que nos ocupa, el arte entró en interlocución con la enfermería, cuyo objeto epistémico centrado en el cuidado de la vida, se abre cada vez con mayor fuerza hacia una investigación de orden interpretativo y comprensivo, que reconoce en las experiencias de los sujetos, individuales y colectivos, la fuente para reinventar formas de cuidado más sensibles y contextualizadas 5 . En este sentido, el encuentro con el lenguaje del arte, enriqueció su campo de indagación y conocimiento, en la medida en que posibilitó superar los límites disciplinares, y abrirse a un campo combinado entre las ciencias sociales y las humanidades en la práctica de investigación.

Concretamente, esta investigación se centró en construir con un grupo de mujeres campesinas, una memoria de sus saberes y prácticas alrededor del alimento, como elemento político nodal en el cuidado de la vida personal, familiar $y$ comunitaria. En articulación con este objetivo, desde la lógica propia del proyecto plástico, se identificó el machete como un objeto icónico, por su fuerte carga simbólica, que permitió construir las memorias en mención desde la metáfora de su doble cara en la trayectoria vital campesina: el envés como arma- objeto de muerte/sufrimiento y el revés como herramienta - objeto de vida/cuidado. 
Teniendo como base este panorama, el artículo pretende resaltar e identificar los puntos sinérgicos entre el proyecto de investigación y el proyecto plástico, cuya relación simbiótica enriqueció la comprensión sensible del conflicto armado, la soberanía alimentaria, la salud mental y la memoria, en un escenario de la ruralidad colombiana con un histórico pasado de despojo y devastación; producto del conflicto armado reciente, a la vez que reconocer la capacidad del arte para integrar y ampliar los diálogos entre saberes diversos y con diversas audiencias.

\section{Materiales y métodos}

El proyecto de investigación se orientó por los presupuestos de la investigación cualitativa, específicamente incorporando elementos de la IAP (Investigación Acción Participativa) ${ }^{6-8}$. Para su desarrollo se contó con la alianza entre una institución académica y una organización campesina, lo cual permitió que más que un proyecto aislado externo, pudiera articularse a procesos de mayor alcance no sólo en el tiempo sino en sus propósitos prácticos sobre la vida local. La conducción de la investigación combinó procesos pedagógicos, con otros de reflexión y de acción en una ruta espiralada de acercamiento y motivación, conformación/preparación de un equipo local de investigación, intercambio de saberes, definición de problemáticas/temáticas particulares y concertación para la producción de la información.

La selección del escenario siguió la lógica de la elección por criterios, consistentes en el acercamiento a territorios de vida de campesinos, pequeños propietarios o jornaleros, en cuya vida se amalgama y potencia el sufrimiento generado por la guerra y la pauperización de una economía tradicional campesina en vía de extinción; sumado a criterios pragmáticos de viabilidad. El muestreo siguió la lógica de la conveniencia, privilegiando la participación de mujeres con un tiempo prolongado de residencia en la localidad seleccionada y con una actividad reconocida en las redes de economía familiar campesina. Para la producción de datos se hicieron entrevistas semiestructuradas (cincuenta) y grupos focales (cuatro), además de quince ejercicios individuales y cuatro ejercicios colectivos de cartografía. También se hizo observación participante de actividades propias de la vida doméstica y de la vida comunitaria relacionada con el trabajo agrícola y se obtuvieron 20 machetes donados por los y las campesinas, los cuales hacían parte de las narrativas y las historias personales, es decir, objetos portadores de memorias diversas. Esta última estrategia surgió como un proceso articulado a la IAP, siguiendo su lógica y orientación.

El proceso estuvo acompañado del registro en diario de campo, además de una amplia documentación audiovisual. El análisis se realizó en forma simultánea con la producción de la información, acogiendo la propuesta de Martí Joel $^{9}$, consistente en una cuádruple lectura: la primera, más inmediata se hizo colectivamente identificando grandes tópicos transversales en relación con los objetivos de la investigación; la segunda focalizó formas de decir, sus sentidos y significados; la tercera se dirigió a indagar por quienes hablan, es decir, los actores sociales desde la posición social que ocupan y el tipo de relaciones en que se encuentran inmersos y finalmente, la cuarta consistió en una aproximación a la síntesis de la información. De esta forma, se logró hacer una reducción de una considerable cantidad de datos, consiguiendo unos procesos de codificación, categorización y síntesis orientados por los propósitos generales del estudio. Se realizó tanto devolución de hallazgos preliminares a las comunidades locales a través de una estrategia de taller comunitario, así como una devolución final mediante asamblea comunitaria con invitación a líderes, representantes de la institucionalidad local y comunidad en general. Esta devolución estuvo además acompañada de la exposición de la obra Memorias sobre machetes, la cual se extendió a diversos escenarios académicos, organizativos y comunitarios.

\section{Consideraciones éticas}

La investigación se condujo bajo criterios de resguardo ético y protección de los participantes y fue avalada por el Comité de Ética de Investigación de la Facultad de Enfermería de la 
Universidad de Antioquia, según consta en el Acta CEI-FE 2014-22 del 2 de mayo de 2014. Entre los resguardos se tramitó el consentimiento para uso de fotografías al inicio de la investigación, ratificándose mediante refrendación del consentimiento, previo al momento de la divulgación final. Se acordó el uso de fotografías y el uso de los nombres grabados en los machetes, en función de los fines académicos de la investigación. Las fotografías que ilustran este artículo son de autoría del equipo de investigación y se utilizan para los fines convenidos con los participantes. El equipo de investigación estuvo integrado por catorce mujeres, cuatro de ellas externas a las comunidades campesinas y las diez restantes miembros activos $y$ residentes en dichas comunidades.

\section{Resultados}

Este trabajo se acercó a la construcción de memorias colectivas en torno al alimento y el trabajo agrícola campesino como generador de cuidado, desde la mirada de las mujeres campesinas. En este sentido la memoria como proceso, se ubicó en el centro de la interacción investigativa con y entre las participantes y se materializó en la obra plástica. Las memorias narradas a través de entrevistas, dibujadas a través de las cartografías e inscritas en el territorio, se incorporaron posteriormente en la materialidad de los machetes, trazando una huella en un objeto con fuerte carga simbólica en tanto tecnología viva de la cotidianidad campesina. (Figura 1).

El proyecto plástico desarrollado en esta experiencia tomó como punto de partida la propuesta de Marcel Duchamp, quien planteaba que el arte debía ir más allá de la representación, tomando objetos de la vida cotidiana que aparentemente no tenían el potencial, ni alcanzaban el estatus de arte, para así cuestionar el arte mismo, convirtiendo dichos objetos en piezas plásticas y acercarse a los sujetos que los producen ${ }^{10}$. En palabras de Doris Salcedo, citada por Cerón ${ }^{11}$ esto implica conectar obra y acontecimiento. Con esta premisa, se incorporó el grabado, técnica que se fundamenta en dejar una huella sobre la superficie de un material, para constituir una memoria objetual, que puede ser percibida con los sentidos y cuya función es reproducir memorias - o copias -, a partir de una matriz o imagen primigenia. Con el fin de concretar ambas pretensiones se introdujo el objeto machete, importado de la realidad a la obra, en tanto elemento familiar capaz de generar un lenguaje simbólico legible, que permitiera a los lectores-espectadores de la obra habitar indirectamente el mundo que representan, por ser este un objeto claramente identificable, conectado con la vida campesina, creíble y verosímil.

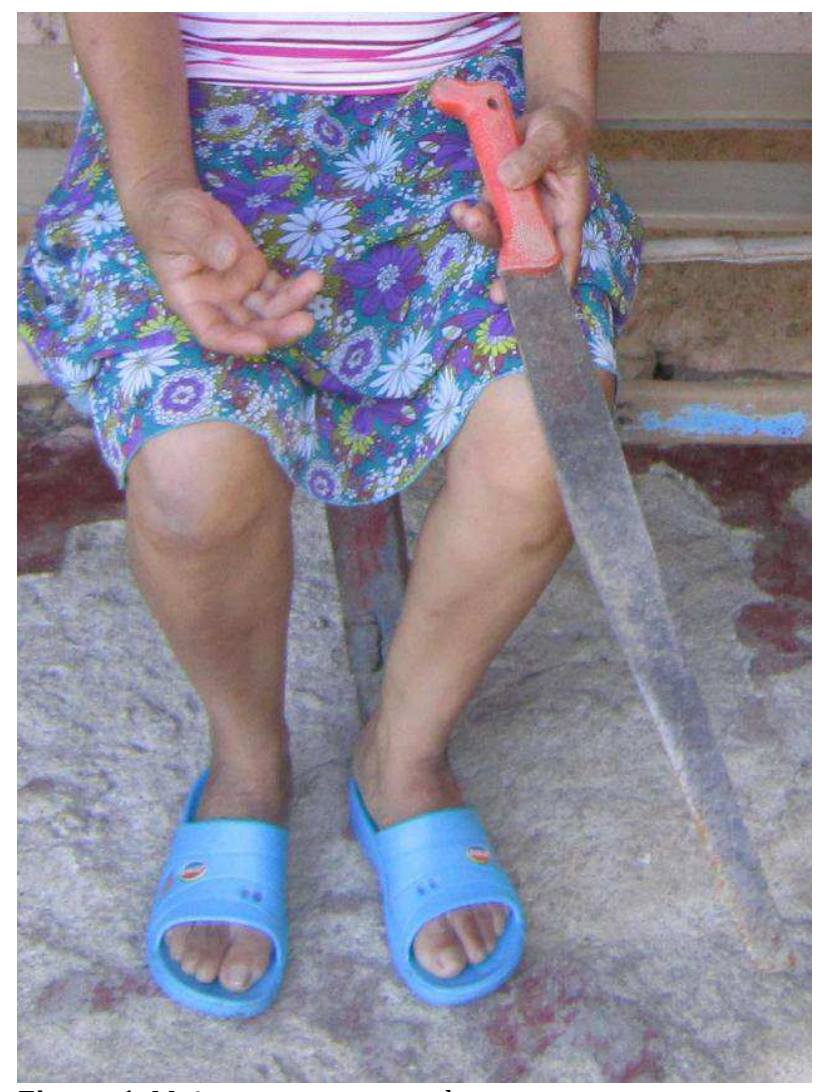

Figura 1. Mujeres, manos y machetes.

Autor: Morganne Blais - McPherson, Memorias sobre Machetes

El machete persiste como la herramienta fundamental del trabajo campesino tradicional, sobre todo en topografías de ladera, se inserta en la distribución de actividades femeninas y masculinas de acuerdo a patrones culturales tradicionales del sistema sexo-género y permea 
las distintas generaciones, desde los niños hasta los más adultos, con usos diferenciales. El machete aparece tanto en las tareas relacionadas con el cuidado de la casa y la familia, incluido el cuidado de las huertas y las especies animales menores, actividades eminentemente femeninas; así como en las labores asociadas a los denominados cultivos "mayores" - café, caña, plátano, frijol, cacao, entre otros - de correspondencia masculina. El uso del machete hace parte de la transmisión oral de un saber práctico que se hereda de generación en generación, como lo afirma una de las participantes: "todos los días uso el machete, prácticamente para todo, es algo vital. Yo no concibo una casa sin un machete, el machete para los campesinos es esencial. Todo campesino su machete tiene que tener. Se usa hasta para la defensa personal".

El machete, toma entonces un lugar privilegiado y consensuado como mediador objetual para plasmar los resultados y facilitar la divulgación de los mismos, articulando el proyecto plástico al proceso metodológico de la investigación. Para ello se obtuvieron machetes usados en la labor agrícola, tanto de hombres como de mujeres, algunos de ellos en desuso por deterioro, que sirvieron de contexto para la inscripción de las biografías personales y comunitarias. El grabado permitió que los machetes que ya contenían memorias propias del tiempo y el uso, fueran re intervenidos con memorias personales, las firmas como primera inscripción, seguidas de diversas narrativas sobre esta herramienta en la vida cotidiana y el trabajo agrícola, fijando en la materialidad tramas de sentido en torno a la producción de alimentos para el cuidado de la vida, la defensa del territorio y el intercambio familiar y vecinal. Los machetes "hablan" de las tensiones propias del contexto global-local, las particularidades culturales del mundo campesino y los procesos emergentes de producción, distribución y consumo de carácter colectivista y agroecológico.

Estos objetos, una vez grabados, fueron expuestos en escenarios públicos de distinta índole, realizando una itinerantica por espacios comunitarios, académicos y organizativos, donde el lenguaje del arte ha potenciado la divulgación de los hallazgos de la investigación a través de la obra Memorias sobre machetes. Los guiones curatoriales han dialogado con los análisis y supuestos de la investigación, manteniendo un intercambio de saberes desde el arte del cuidado que pretende la enfermería y las artes plásticas en su concreción y materialidad.

Para ello se puso en la escena pública una instalación de 118 objetos: algunos son machetes usados y donados por los y las campesinas, grabados con su firma personal y con sus testimonios, como recurso objetual que reconoce los sujetos campesinos en su dignidad y resistencia (Figura 2).

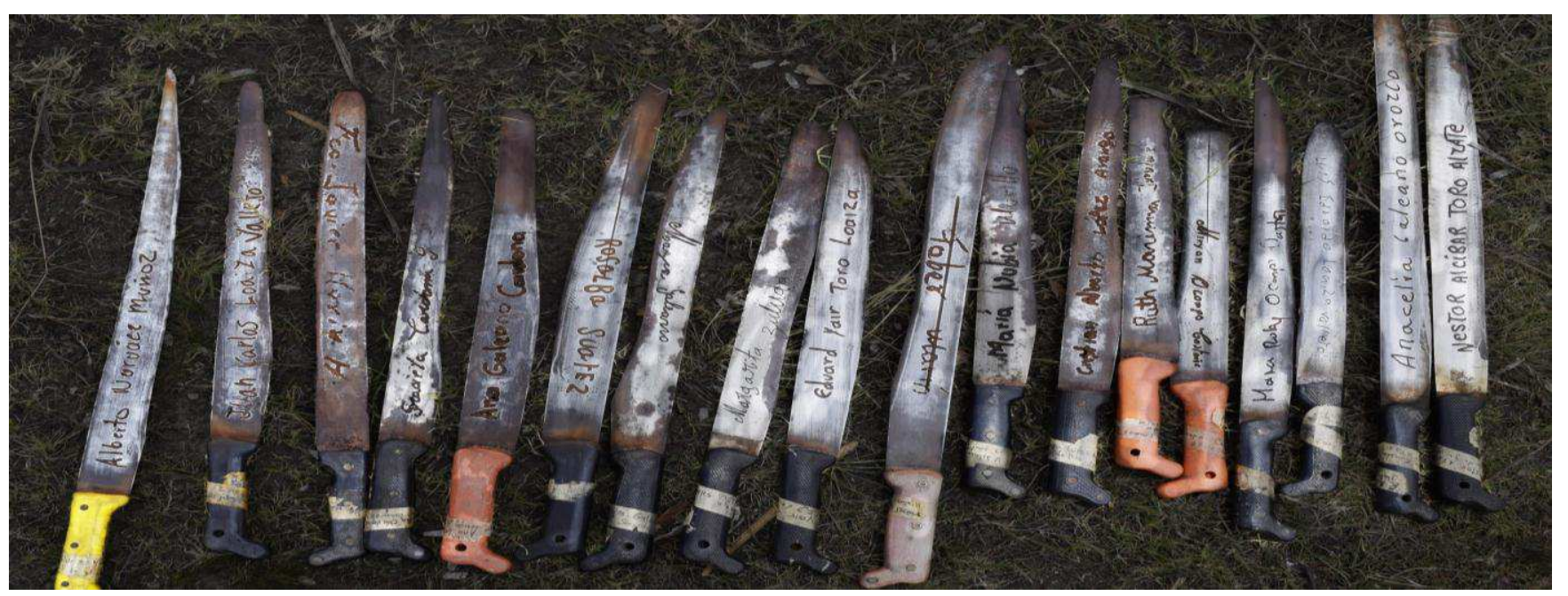

Figura 2. Machetes y nombre propio.

Autor: Laura Antonia Coral. Archivo Memorias sobre Machetes 
De otro lado, machetes grabados con los nombres de ríos que señalan lugares de muerte documentados en la historia del conflicto colombiano, en los cuales este objeto se usó como arma (Figura 3). Los primeros se instalan sobre la tierra, algunos clavados verticalmente para simbolizar la permanencia "en pie" de los y las campesinas en sus territorios (Figura 4), mientras los segundos flotan sobre espejos de agua, simbolizando los cuerpos caídos en la guerra.

La pretensión es compartir con el espectador una de las conclusiones del estudio en relación con esta memoria colectiva que reconoce que, a pesar del conflicto, los y las campesinas conservan un espacio de soberanía, en la medida en que persisten en su empeño por obtener de la tierra lo necesario para sobrevivir, y que es posible aún emprender proyectos colectivos que les permiten subsistir y reinventar su existencia.

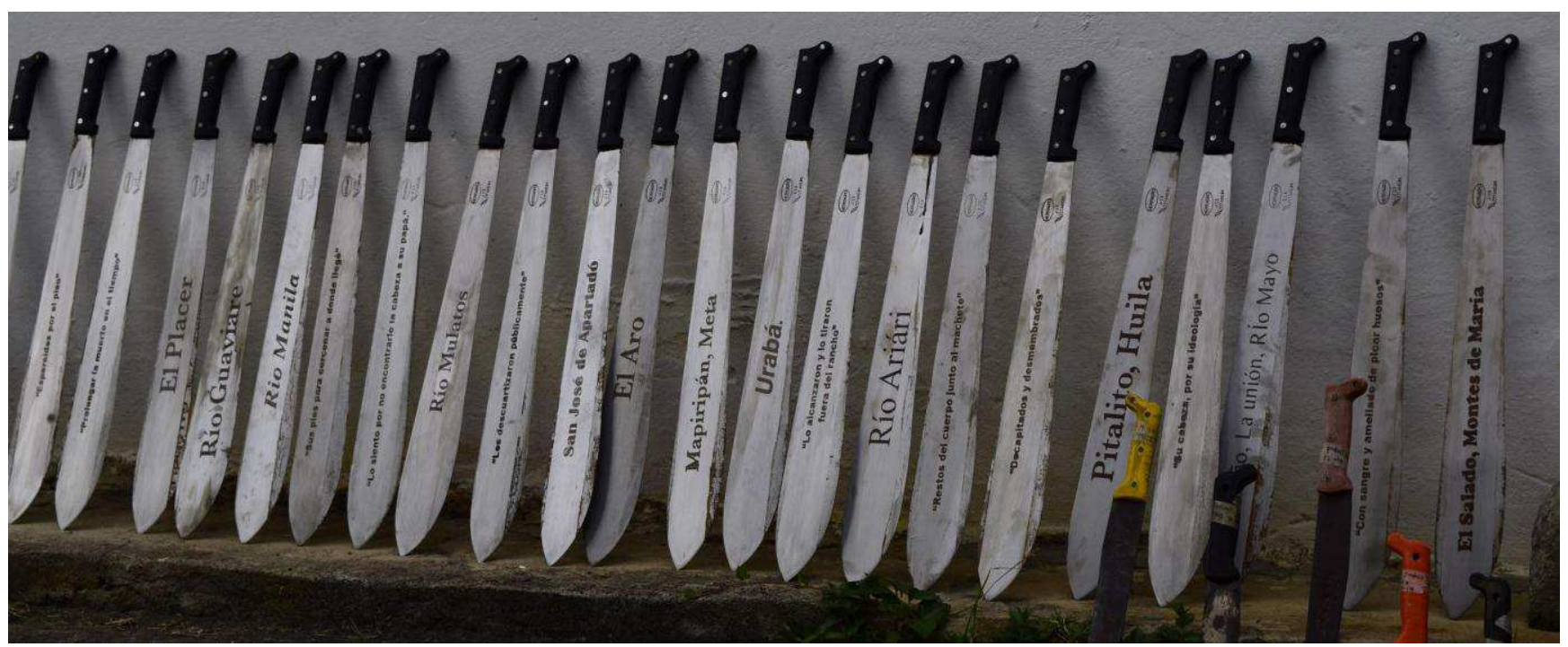

Figura 3. Machetes y memorias.

Autor: Laura Antonia Coral. Archivo Memorias sobre Machetes

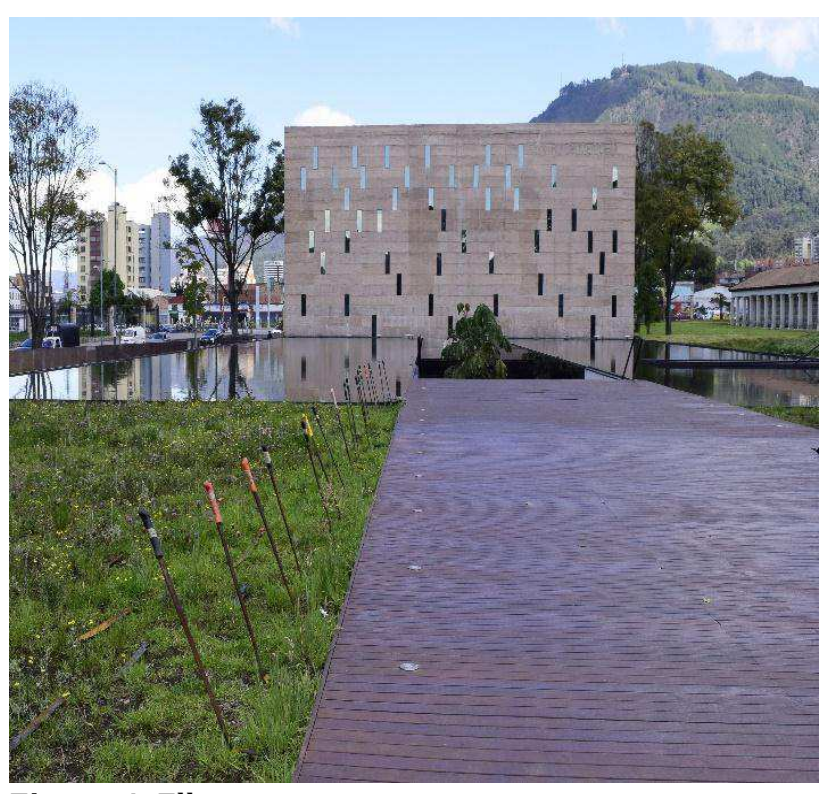

Figura 4. Ellos resisten.

Autor: Laura Antonia Coral. Archivo Memorias sobre Machetes
Estos espacios de difusión permiten el surgimiento de otras memorias a partir de la reflexión con los espectadores, quienes interpelados por el lenguaje del arte, amplían las narrativas del machete como símbolo de soberanía campesina y alimentaria en la historia reciente, personal y colectiva, en un país cuyos lazos comunes con la vida campesina son espacial y temporalmente cercanos. La imagen del machete grabado con la firma de un campesino, es recibida como veraz, auténtica y próxima por quien observa la obra, generando reconocimiento y solidaridad con dicho sujeto, como uno de los alcances éticos más importantes.

\section{Discusión}


La experiencia generó en las participantes, especialmente en las investigadoras externas a la comunidad, una serie de rupturas con algunos presupuestos disciplinares y prácticos que debieron ser de construidos y re aprendidos en el proceso. Una de ellas fue la ruptura con el esquema del artista y el investigador "culto y erudito", para involucrar a las participantes en el proceso creativo - investigativo, en correspondencia con los supuestos praxiológicos de la IAP, reconociendo en la interacción con el equipo de coinvestigadoras locales, mujeres campesinas residentes en la zona rural, la posibilidad de un acercamiento investigativo más sensible, derivado de la interacción dinámica entre lo emocional y lo cognitivo, favorecido en forma significativa por la mediación artística.

La construcción de memorias, por su parte se problematizó, incorporando los debates entre una memoria hegemónica preponderante, frente a la convergencia/divergencia de diversas memorias, lo que implicó la singularidad en el proceso de grabado, cuya expresión por excelencia fue la firma de los participantes, rompiendo así con la lógica de la reproducción y la copia del grabado. La firma se constituye en un acto soberano de afirmación propia, cuya posibilidad a través del arte expande el autoconocimiento y el conocimiento de los demás. Los machetes firmados y grabados de su puño y letra constituyen una memoria tangible de resistencia y soberanía, ya que la firma como representación gráfica, no sólo presenta el nombre, sino que simboliza la persona viva que existe detrás de cada trazo y de una fuente tipográfica irrepetible.

El proyecto artístico Memorias Sobre Machetes incorpora los hallazgos, optimizando su valor comunicativo y la creación de oportunidades para el compromiso de las participantes en todas las fases del proceso de investigación. La sensibilidad que permite el arte en su carácter mediador, expandió las posibilidades de acercamiento con los sujetos, aumentando el interés por investigar y flexibilizó las formas tradicionales para construir la multiplicidad de memorias, que, sin perder su particularidad, confluyeron en torno al objeto plástico. La confluencia de diversos testimonios en este objeto, buscó un potencial diálogo con cualquier espectador, instaurando la obra final como un puente potente de comunicación en otros escenarios, incluso distantes, espacial y culturalmente, del que le dio origen a la obra.

Los hallazgos convertidos en arte, se pudieron difundir de forma privilegiada ${ }^{12}$ dada la posibilidad para las participantes de la investigación de ser activas en el relato e inscripción de su propia historia y posteriormente, a través de las exposiciones, mostrarse a otros en una suerte de proclama individual y colectiva, grabada en un objeto de fuerte carga simbólica. Por su parte los testigos externos, han contribuido al reconocimiento y autenticación de las afirmaciones de aquellos, otorgando carácter público y fáctico a las mismas y por lo tanto legitimidad social. De esta forma, la divulgación de los resultados de la investigación a través del arte constituye una especie de ceremonia de definición compartida ${ }^{13}$, en la medida en que instaura un escenario autorreflexivo que permite a las personas ser conscientes del papel productor de sus propias vidas y sentirse agentes activos en ellas.

Si bien no fue una intención explícita, los resultados de esta experiencia se aproximan a los debates de un movimiento que emergió en los años setenta del siglo pasado en el mundo anglosajón bajo la denominación de Investigación basada en el arte, movimiento que adapta las tendencias de las artes creativas en la investigación social para hacer la investigación públicamente accesible, evocativa y comprometida, mediante la convergencia sinérgica entre formas artísticas de expresión y el proceso científico. Según Chilton y Leavy ${ }^{14}$ en la investigación en salud se ha documentado ampliamente que las artes son útiles para descubrir, producir y difundir conocimiento, con fuerte aporte para liderar el cambio social, dado su potencial para generar estrategias participativas efectivas, que pueden derivar incluso en el desarrollo de políticas públicas.

Finley ${ }^{15}$ propone repensar la utilidad de las artes cuando la finalidad de la investigación es el activismo político, en términos de su utilidad 
social y sus metodologías. Estas discusiones de reciente ingreso en el contexto colombiano, se asoman tímidamente en estos resultados, pero sin duda constituyen vías promisorias para continuar avanzando en estos diálogos transdisciplinares de índole tanto epistemológica, como política y ética.

Concretamente en esta experiencia se produjo un diálogo entre la enfermería y las artes plásticas, posibilitando a la primera fortalecer la investigación sobre el cuidado desde su dimensión estética y comunicativa, superando la mirada sobre acciones instrumentales prescriptivas, que aíslan y no permiten el entendimiento, ni la solidaridad con los sujetos de cuidado $^{16}$ contribuyendo en su lugar a comprender versiones particulares de las mujeres campesinas, desde sus contextos de vida.

En ambos campos disciplinares, las artes y la enfermería, se suscitaron preguntas sobre el papel de los conocimientos académicos y los saberes "cultos" y la manera cómo se imbrican las lógicas intelectuales con las lógicas vitales ${ }^{17}$, al amalgamar intenciones profundamente existenciales que surgen de las biografías personales de las investigadoras, las orientaciones teóricas disciplinares y el contexto espacial e histórico específico.

Uno de los grandes aportes a la consolidación de una estética del cuidado, tuvo que ver con la propuesta artística de relevar el carácter significativo de un objeto como el machete y desde esa sensibilidad resignificar la experiencia de las participantes. La emocionalidad involucrada en el proceso no solo se circunscribe al objeto en sí mismo, sino que habla de la expresión de una belleza implicada en el mundo de las relaciones y en los sujetos involucrados, expresando de esa manera una sensibilidad: por un lado al reconocer un Otro en la relación de cuidado; en segundo lugar al permitir que las investigadoras se situaran en la perspectiva de sujetos implicados y finalmente al acercar a múltiples Otros, a través de la puesta en escena pública de la obra plástica. Como afirma Pozzoli 18 "el arte puede definirse como una vía de expresión de nuestra conciencia, y como un ámbito de la expresión y de la creación humana en el que podemos reconstituir, expresar o reconocer nuestra interioridad" posibilidad que se extendió tanto a participantes, investigadoras $\mathrm{y}$ otros espectadores de la obra.

Particularmente estos aportes contribuyen a fortalecer el llamado patrón estético de conocimiento en enfermería, a partir de una experiencia concreta que involucra la co-creación y apreciación de una realidad única y singular, bajo la intención explícita por comprender los significados en las relaciones sociales del contexto campesino y la posibilidad de establecer encuentros significativos con las personas participantes. Para Duran ${ }^{19}$ esto implica “imaginar posibilidades y formar elementos que permitan percibir la realidad a través de representaciones creativas con posibilidades de transformar el comportamiento de los pacientes y de la enfermera", en dirección a la búsqueda de sentidos, a través de la sensibilidad que permiten el arte y la belleza.

Esta experiencia, como otras ya documentadas 20,21 , se interesó particularmente por escuchar las voces de sujetos que socialmente han estado ubicados en las márgenes o las periferias del sistema económico y social, en tanto campesinos pobres, que habitan territorios históricamente excluidos y despojados y cuya viabilidad existencial ha sido puesta en tela de juicio. La articulación entre la propuesta investigativa y plástica, tuvo como intención política y ética, la aproximación al compromiso con la "verdad" y "significado" por fuera de jerarquías verticales, interpelando no sólo los modelos de desarrollo rural que están en debate en el país, sino además las lógicas hegemónicas de construcción de conocimiento. En este caso la participación activa de las mujeres en la investigación opera en favor de una lógica más "horizontal", claramente expresada en la obra plástica, que tiene efectos de orden práctico en la vida local y personal, más allá de las discusiones teóricas que muchas veces cursan por fuera de los circuitos culturales que les dan origen.

\section{Conclusiones}


En este artículo hemos presentado una experiencia de investigación cualitativa en torno al cuidado comunitario en un contexto rural con una historia de conflicto armado, exclusión social y pobreza, en la cual se integraron elementos de la investigación participativa con el desarrollo de un proyecto plástico, realizado con mujeres de una zona campesina del oriente antioqueño colombiano. La experiencia deja algunos aprendizajes significativos desde el punto de vista epistemológico, ético y político, relacionados con el fortalecimiento del diálogo transdiciplinar entre las artes y la enfermería; con el fortalecimiento de la investigación cualitativa desde los aportes del arte; con la potenciación de las voces que se encuentran en las márgenes o la periferia del sistema social y con la incorporación del arte como estrategia potente para la construcción de memorias colectivas.

La investigación cualitativa, al igual que las artes, comparten la intención y posibilidad del acercamiento profundo a los fenómenos relacionados con la existencia humana, facilitando una aproximación sensible a las realidades subjetivas. Los procedimientos de la técnica o de la creación artística pueden insertarse en la investigación cualitativa tanto para producir datos, analizarlos, representarlos o lograr múltiples variaciones y combinaciones, en una lógica no lineal, que estimula a los participantes a generar nuevas reflexiones. En el contexto de la investigación cualitativa, las artes materializan una experiencia viva que integra la sensibilidad y los procesos sociales para crear una imagen que ayuda a entender la vida de quien participa en su construcción y que puede proyectarse hacia otros, descartando la postura del artista iluminado y más bien ofreciendo la oportunidad de creación al investigador y de investigación al artista.

Memorias sobre machetes transformó en materialidad las reflexiones suscitadas en el proceso investigativo y creativo, permitiendo un mayor acercamiento intersubjetivo y otorgando herramientas de comprensión colectivas e individuales, para comunicar historias y trazar recorridos. Apelar a materiales u objetos situados significativamente en las trayectorias vitales- sociales de las personas y comunidades, como se hizo en este caso, hace posible que la construcción de memorias, la investigación y la contextualización de los cuidados se tornen en procesos creativos que orientan formas de construcción colectiva de conocimientos con fuertes compromisos éticos y políticos.

\section{Recomendaciones}

La experiencia y los análisis presentados señalan la necesidad de ahondar en una revisión sistemática que permita reconocer la sinergia entre experiencias de investigación en salud en su encuentro con las artes, en sus múltiples manifestaciones. Esta exploración es aún precaria en el contexto nacional y latinoamericano, encontrando sobre todo trabajos del mundo anglosajón, que dejan este como un camino por explorar. Desentrañar y comprender las lógicas, supuestos y procedimientos del arte en el contexto investigativo, conducirá a comprender las particularidades del conocimiento que de allí emerge, especialmente en el campo de la salud y por ende su legitimidad en relación con el acercamiento a los espacios íntimos y las subjetividades.

\section{Agradecimientos}

A las campesinas del municipio de Argelia por su hospitalidad y su apertura para compartir sus conocimientos.

A las compañeras del equipo investigador Luz Estella Cifuentes y Elsa Pilar Parra Mojica (Asociación Campesina de Antioquia ACA), Ana Galeano Cardona, Diana María Arango Dávila, Marina Galeano, Luz Myriam Ocampo Castaño, María Rubí Ocampo Castaño, Ana Celia Galeano Orozco, Jaqueline Zuluaga, Henry Zuluaga, Leidy Narváez, Luz Dey Narváez y Heidy Narváez (Equipo local de investigadoras campesinas); Estudiante en pasantía Morganne Blais McPherson (Universidad Mc Guill) A la Facultad de Enfermería de la Universidad de Antioquia y a la Asociación Campesina de Antioquia ACA por su apoyo para la realización de este proyecto.

Conflicto de intereses: Ninguno declarado por los autores. 


\section{Referencias}

1. Leavy P. Method meets art: Arts-based research practice. New York: Guilford press; 2009.

2. Barone T, Eisner E. Arts Based Research. Los Angeles: Sage; 2012.

3. Rech L. Arts Based Research. Studies In Art Education. 2013;55.

4. Hernández F. La investigación basada en las artes. Propuestas para repensar la investigación en educación. Educatio Siglo XXI. 2008;26:85-118.

5. Beck Ch. Routledge international handbook of qualitative nursing research. New York: Routledge; 2013.

6. Fals Borda 0. Reflexiones sobre investigación acción participativa. Medellín: Universidad de Antioquia; 1985.

7. Abad DP. La investigación-acción-participativa: una forma de investigar en enfermería. Investigación y Educación en Enfermería. 2010;28:464-74.

8. Ander Egg E. Repensando la IAP. Buenos Aires: Lumen Hvmanitas; 2003.

9. Martí J. Investigación acción participativa, estructura y fases. En: La investigación social participativa. Madrid: El Viejo Topo; 2002. p. 79-123.

10. Calvesi M. Duchamp. Barcelona: Planeta D'Agostini; 1999.

11. Cerón J. Memoria y dolor, el contramonumento de Doris Salcedo. Semana [Internet]. 13 de octubre de 2002; Disponible en: http://www.semana.com/cultura/articulo/memoriadolor contramonumento-doris salcedo/54519-3

12. Boydell KM, Gladstone BM, Volpe T, Allemang B, Stasiulis, E. The production and dissemination of knowledge: A scoping review of arts-based health research. Forum Qualitative Sozialforchung/Forum: Qualitative Social Research [Internet]. 2012;13. Disponible en: http://nbnresolving.de/urn:nbn:de:0114-fqs1201327

13. White M. Reescribir la vida. Barcelona: Gedisa; 2002.

14. Chilton G, Leavy P. Arts-Based Research Practice: Merging Social Research and the Creative Arts. En: The Oxford Handbook of Qualitative Research. New York: Oxford University Psychology press; 2014. p. 403-22.

15. Finley S. Arts-based inquuiry. Performing revolutionary pedagogy. En: The sage handbook of quialitative research. California: Sage; 2008. p. 680-94.

16. Duque M. Solidaridad y ciencia. Reflexiones en el contexto del cuidado de enfermería como interacción comunicativa. Investig Enferm Imagen Desarro. 2011;13:101-12.

17. Castillejo A. Utopía y desarraigo. Papeles del CEIC [Internet]. 2015;1. Disponible en: http://dx.doi.org/10.1387/pceic.130362015

18. Pozzoli MT. Espiritualidad, arte y belleza. Espacios del universo para el desarrollo humano desde el pensamiento complejo. Polis [Internet]. 2007;17. Disponible en: http://polis.revues.org/4305
19. Duran MM. La ciencia, la ética y el arte de enfermería a partir del conocimiento personal. Aquichán. 2005;5:8695.

20. Smit A. Everyone but Rizzo: Using the Arts to Transform Communities. Forum on Public Policy. 2008;2:1-21.

21. Arias BE. Hand-Woven Narratives of Peasant Resistance. Three questions about a Colombian experience. En: Peace Building, Gender and Social Work. Núremberg: Paulo Freire Verlang Oldenburg; 2015. p. 247-60. 